\title{
Target Tracker detector of the OPERA experiment
}

\section{Sergey Dmitrievsky ${ }^{1}$ on behalf of the OPERA Collaboration}

Joint Institute for Nuclear Research

Joliot-Curie 6, 141980 Dubna, Moscow region, Russia

E-mail: dmitrejinr.ru

Target Tracker (TT) is the most important electronic detector of the hybrid setup of the OPERA experiment. Built of 32000 plastic scintillator strips, this detector is a main tool for the neutrino interaction registration in real time and for the vertex location for the further detailed analysis of the interaction in nuclear photoemulsion. It also provides a neutrino interaction trigger for the readout of the whole detector, and is used as a calorimeter for the event analysis. High detection efficiency ( $\sim 99 \%$ for a mip) is stable since TT installation in 2006 , no significant ageing is observed. Here we present a description of the detector, its main characteristics, methods and algorithms used for the data processing, discuss a performance of the detector for the vertex location.

Speaker 


\section{Introduction}

The OPERA experiment [1] is designed for the first direct observation of $v_{\tau}$ appearance from $v_{\mu} \rightarrow v_{\tau}$ oscillation in the CNGS [2] long baseline beam from CERN to the underground Gran Sasso Laboratory (LNGS), $730 \mathrm{~km}$ away, where the OPERA detector is located. The challenge of the OPERA is the detection of the short-lived $\tau$ lepton $(\mathrm{c} \tau=87 \mu \mathrm{m})$ produced in the charged-current $(\mathrm{CC})$ interaction of $v_{\tau}$. This sets two requirements difficult to conciliate: a large target mass to collect enough statistics and a very high spatial accuracy to identify the $\tau$ via the topological observation of its decay. OPERA hybrid setup combines real-time detection techniques ("electronic detectors") and the Emulsion Cloud Chamber (ECC) technique. The target part of the detector contains lead/emulsion modules (called bricks), for a total mass of $\sim 1.25 \mathrm{kton}$, arranged in walls interleaved with planes of plastic scintillator strips that constitute Target Tracker (TT) [3]. TT is a main tool for the neutrino interaction registration in real time and for the vertex location for the further detailed analysis of the interaction in nuclear emulsion. It also provides a neutrino interaction trigger for the readout of the whole detector, and is used as a calorimeter for the event analysis.

\subsection{Target Tracker overview}

Each of 62 TT planes is composed of a pair of orthogonal arrays of 256 scintillator strips $6.86 \mathrm{~m}$ long, $1 \mathrm{~cm}$ thick, and $2.6 \mathrm{~cm}$ wide, read on both sides using Wavelength Shifting fibres and multi-anode photomultipliers (PMT). The particle detection principle is depicted by Fig. 1.

Before TT assembly all scintillator strips had been tested and calibrated with electrons from a

${ }^{90} \mathrm{Sr} \beta$ source simulating energy lost by a minimum ionizing particle (mip) in $1 \mathrm{~cm}$ of plastic scintillator. The mean number of photoelectrons (p.e.) observed at the middle of the scintillator strips (i.e. the worst position for light collection) was found to be 5.9 p.e. Mean detection efficiency

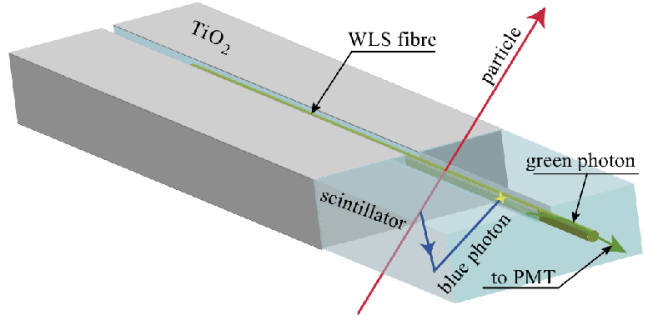

Fig. 1. Parlicle delection principle in a scintillating strip. for a mip crossing the strip at the middle was higher than $99 \%$ for discriminator threshold corresponding to $1 / 3$ p.e., while the background trigger rate (dark current, radioactivity, etc.) stayed below $20 \mathrm{~Hz}$.

The obtained parameters of attenuation of the signal along the strip fibre are used to reconstruct energy deposition when detecting neutrino events. During OPERA data taking TT detection efficiency and ageing of plastic scintillator were monitored using cosmic ray data. Analysis of $\sim 1.4$ million collected 3D muon tracks showed stable TT detection efficiency at the level of $98 \%$ for 1 p.e. threshold and ageing of plastic scintillator at the level of $0.7 \%$ year.

\section{Localization of the neutrino interaction vertex}

The most important task of the TT is a "brick finding" (BF) - localization of the ECC brick that contains a primary neutrino interaction vertex [4]. After this primary localization, a further 
search of the vertex inside the brick is performed by the emulsion analysis methods. High BF efficiency allows to reduce emulsion scanning load and to minimize the target mass loss.

Analysis of the electronic detectors data for the brick finding includes several steps briefly described below.

1) Primary processing of the TT hits. At this step energy deposition of each hit is estimated from the measured PMT amplitudes at both ends of the corresponding TT strip. Then isolated hits or small groups of hits (that are usually present in the detector due to natural radioactivity background, PMT noise and channels cross-talks, etc.) are suppressed in order to make easier track reconstruction. For this purpose event filtration procedure based on the cellular automaton approach [5] is applied.

2) Muon track reconstruction. In general case, the TT resolution $(\sim 7.5 \mathrm{~mm})$ doesn't allow distinguishing tracks in a shower near the interaction vertex. Nevertheless, muons produced in charged current neutrino events $\left(v_{\mu} \mathrm{CC}\right)$, as well as in $\tau \rightarrow \mu$ events, are usually have long tracks and can directly indicate the brick where they are originated from. Two algorithms are applied in the $\mathrm{BF}$ procedure for the muon track reconstruction: the one based on the Hough transform [6] (for reconstruction of straight tracks) and so-called track-following method (for reconstruction of curved tracks).

3) Reconstruction of a hadronic shower axis. In many cases, like, for instance, in the neutrino NC interaction events, there are no clear tracks to point to the vertex. In those cases, a reconstruction of the hadronic shower axis is used for the vertex brick finding. Due to the fact that the energy deposition in the TT along the direction of shower development is supposed to be higher, the axis can be reconstructed taking into account positions of the hits weighted by their amplitude. This is realized in method of robust line fitting [7].

4) Determination of the vertex wall candidates. Difficulty of the vertex wall determination is mainly related to presence of back-scattering particles. These particles leave signals in the TT planes upstream of the vertex brick extending the search area. In order to recognize the vertex wall among first several hit walls a Multilayer Perceptron (MLP) [8] is used. It performs analysis of signals in three selected TT planes and estimates probabilities for the corresponding target walls to contain the vertex brick. Values used as input parameters for the MLP are number of hits, deposited energy, dispersion of the hit density, etc.

5) Prediction of the vertex brick candidates. After the reconstruction of the muon track and the hadronic shower axis, their parameters as well as the MLP output are used for identification of a set of bricks that most likely contain a neutrino interaction vertex. Calculation of the brick probabilities takes into account the real positions of all bricks inside the detector at the time of event registration.

Described BF procedure is implemented as an open-source software package integrated in the OPERA data processing framework. Tuning of the algorithms was performed on simulated data. The BF efficiency is estimated to be $65 \%$ for 2010-2012 OPERA data sample.

\section{Measurement of the neutrino velocity}

In addition to its main goal, OPERA experiment is well suited to determine the neutrino velocity with high accuracy through the measurement of the time of flight (TOF) and of the 
distance between the source of neutrinos and the detector. Dedicated upgrades of the timing systems for the time tagging and synchronization based on GPS technique resulted in a reduction of the systematic uncertainties down to the level of the statistical error. The measurements also rely on a geodesy campaign that allowed measuring the $730 \mathrm{~km}$ CNGS baseline with a precision of $20 \mathrm{~cm}$.

In 2011 OPERA reported the observation of an anomalous value of the muon neutrino TOF which could be interpreted in terms of superluminal propagation. Continuing carefully to scrutinize the measurement, two unaccounted systematic effects significantly affecting the result were found by the Collaboration. A final result taking into account those effects showed no significant difference between the speed of light and the neutrino velocity [9].

In order to cross-check the revised result and increase the accuracy the measurement of the neutrino TOF at the single interaction level with a narrow bunch proton beam (BB) was performed in May of 2012. Target Tracker detector was used for independent measurement of the neutrino arrival time [10]. TT data were analyzed by two methods. The first method relied on the information of the earliest hit, while the second one exploited the time information of all hits of the reconstructed 3D muon track providing better time resolution for CC-like events.

The final combined result for the deviation of the muon neutrino velocity from the speed of light is $(0.6 \pm 0.4($ stat.$) \pm 3.0($ syst. $)) \mathrm{ns}$, giving the limit $-1.8 \times 10^{-6}<\left(\mathrm{v}_{v}-c\right) / c<2.3 \times 10^{-6}$ at $90 \%$ C.L.

\section{References}

[1] M. Guler et al. An appearance experiment to search for $v_{\mu} \rightarrow v_{\tau}$ oscillations in the CNGS beam: experimental proposal, CERN-SPSC-2000-028, LNGS P25/2000

[2] Ed. K. Elsener, The CERN Neutrino beam to Gran Sasso (Conceptual Technical Design), CERN 98-02, INFN/AE-98/05

[3] T. Adam et al, The OPERA experiment Target Tracker, NIMA 577 (2007) 523-539

[4] A. Chukanov et al., Neutrino interaction vertex location with the OPERA electronic detectors, 2013, OPERA note n.162. Available at http://operaweb.lngs.infn.it/Opera/publicnotes/note162.pdf.

[5] A.Glazov et al, Filtering Tracks in discrete detectors using a cellular automaton, Nucl. Instr. and Methods, A-329, (1993), 262-268

[6] V.C.Hough, A Method and Means for Recognizing Complex Patterns, US Patent: 3, 069, 654, Dec. 1962

[7] G.A.Ososkov, Elastic Arm methods of Data Analysis as a Robust Approach, Tatra Mount. Math.Publ., 26 (2003), 291-306

[8] T.Khanna, Foundation of Neural Networks, N.Y.: Addison-Wesley (1989)

[9] T. Adam et al, Measurement of the neutrino velocity with the OPERA detector in the CNGS beam, JHEP 10 (2012) 093

[10] T. Adam et al, Measurement of the neutrino velocity with the OPERA detector in the CNGS beam using the 2012 dedicated data, JHEP 01 (2013) 153 